Document downloaded from:

http://hdl.handle.net/10251/52669

This paper must be cited as:

López-Mir, F.; Naranjo Ornedo, V.; Angulo, J.; Alcañiz Raya, ML.; Luna, L. (2014). Liver segmentation in MRI: a fully automatic method based on stochastic partitions. Computer Methods and Programs in Biomedicine. 114(1):11-28. doi:10.1016/j.cmpb.2013.12.022.

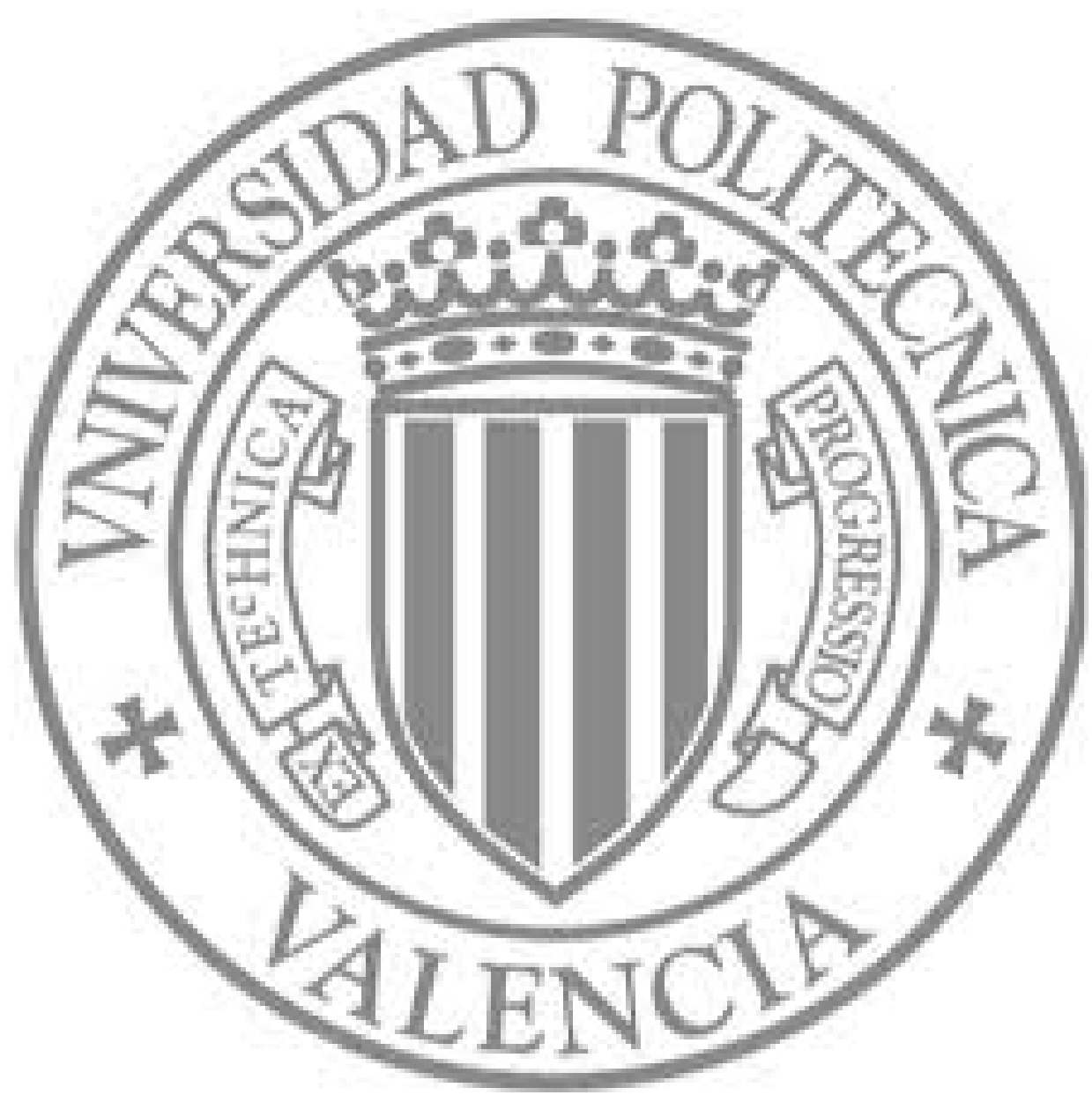

The final publication is available at

http://dx.doi.org/10.1016/j.cmpb.2013.12.022

Copyright Elsevier 


\title{
Liver segmentation in MRI: A Fully automatic Method Based on Stochastic Partitions
}

\author{
F. López-Mir ${ }^{\mathrm{a}, *}$, V.Naranjo $^{\mathrm{a}}$, J. Angulo ${ }^{\mathrm{b}}$, M. Alcañiz ${ }^{\mathrm{a}, \mathrm{d}}$, L. Luna ${ }^{\mathrm{c}}$, \\ ${ }^{a}$ Instituto Interuniversitario de Investigación en Bioingeniería y Tecnología Orientada al \\ Ser Humano, Universitat Politècnica de València, Camino de Vera s/n, 46022, Valencia, \\ Spain. \\ ${ }^{b}$ Centre de Morphologie Mathématique, Mathématiques et Systèmes, MINES Paristech, \\ France \\ ${ }^{c}$ Hospital Clínica Benidorm (Unidad de Resonancia Magnética INSCANNER), Spain \\ ${ }^{d}$ Ciber, Fisiopatología de Obesidad y Nutrición, CB06/03 Instituto de Salud Carlos III, \\ Spain
}

\begin{abstract}
There are few fully automated methods for liver segmentation in magnetic resonance images (MRI) despite the benefits of this type of acquisition in comparison to other radiology techniques such as computed tomography (CT). Motivated by medical requirements, liver segmentation in MRI has been carried out. For this purpose, we present a new method for liver segmentation based on the watershed transform and stochastic partitions. The classical watershed over-segmentation is reduced using a marker-controlled algorithm. To improve accuracy of selected contours, the gradient of the original image is successfully enhanced by applying a new variant of stochastic watershed. Moreover, a final classifier is performed in order to obtain the final liver mask. Optimal parameters of the method are tuned using a training dataset and then they are applied to the rest of studies (17 datasets). The obtained results (a Jaccard coefficient of .91 \pm .02 ) in comparison to other methods demonstrate that the new variant of stochastic watershed is a robust tool for automatic segmentation of the liver in MRI.

Keywords: Magnetic resonance imaging; liver segmentation; mathematical morphology; stochastic partitions; watershed transform.
\end{abstract}

\footnotetext{
* Corresponding author

Email address: ferlomir@labhuman.i3bh.es (F. López-Mir)
}

Preprint submitted to Computer Methods and Programs in Biomedicine December 20, 2013 


\section{Introduction}

Fully automatic liver segmentation in medical images is currently an unsolved problem [1]. An accurate liver segmentation has a direct application in the planning, monitoring, and treatment of different types of pathologies such as cirrhosis or hepatocellular carcinoma diseases. In these cases, hepatic tissue anomalies are treated using qualitative comparison, which is related to physician experience; however, quantitative measures are not widely used. Liver segmentation is the first step to calculate objective measurements and liver/lesion ratios for decisions regarding treatment and planning for the patient. The segmentation of internal organs is also essential for image-guided surgery and virtual reality scenarios for medical training $[2,3,4,5,6]$. In addition, the liver segmentation can help in hepatic steatosis quantification because the results of this segmentation can be correlated to measure fat fractions [7].

In most applications mentioned above, due to the high accuracy required, a segmentation of the liver is carried out in images with high spatial resolution, i.e., Computed Tomography (CT) or Magnetic Resonance Images (MRI) [8, 9]. Currently, some efforts are focused on the segmentation of the liver in other types of images (such as PET or ultrasound images) that are less damaging for the patient than the CT images and that are cheaper for hospitals than MRI. However, the low spatial resolution of these images is a disadvantage and, in some cases, even with manual corrections, the segmentation is not accurate enough for image-guided surgery or liver volumetry applications that radiologists or surgeons require $[10,11]$. In the literature, there are more segmentation methods that are applied and validated for CT than for MRI. MRI generally has more artefact effects and a lower gradient response and is more costly for hospitals; however, since it is a non-ionizing radiation, it is less damaging for the patient in comparison with CT. The authors of several studies support the benefits (or the additional information) of MRI considering it to be a primary diagnostic imaging modality for liver lesion detection or for measuring hepatic 
steatosis $[7,12,13,14,15,16]$. For example, the segmentation of the liver in MRI is important in automating liver perfusion analysis, which provides important information about the blood supply to the liver [17]. In any case, hepatic MR certainly is an alternative to CT images for the diagnosis of liver disease offering benefits that make this image technique interesting for clinical purposes. For this reason it is necessary to advance in the development of methods for liver segmentation in MRI in a way similar to the advances in CT methods.

The liver segmentation methods found in the state-of-the-art in MRI are based mainly on level-set methods $[17,18,19,20,21,22]$, where the drawbacks of these algorithms (difficult training, high computational cost, or high user iteration) are noticeable. Specially, in [18], a level-set method (a fast marching algorithm) and fuzzy theory are applied in the liver segmentation task, but the computational cost of this algorithm needs to be improved (as the authors themselves recognize) and, additionally, non-uniform intensity problems are not solved. In [19, 20], level-sets and probabilistic maps are used, and a training process is required with a high user iteration for manual segmentation. In [21], another level-set method called active contour is applied in T1 MR images of the liver, and the radiologist's knowledge is required to define the region of interest. Finally, in [22], active contours are also applied in T2 MR images, but the results, though promising, are not accurate in some cases. Other methods based on graylevel properties (region growing, thresholding, k-means, etc) produce poor results in images of this type because the great intra-study differences make difficult the generalization of these algorithms. In [9], authors use a region growing method combined with threshold techniques and prior knowledge that requires a training step with manual segmentation.

In the current paper, the performance and the validation of a new liver segmentation method that is based on the watershed transform and that is applied to MRI is presented. The goal is to obtain a fully automatic method that requires less of the clinician's time, has enough accuracy and robustness for medical environments, and has a reasonable computational cost. The watershed transform is a segmentation tool that is based on graylevel and contour 
properties of the image. This tool extends each regional minimum of the image as far as its topography allows. An over-segmentation problem usually appears due to the large number of regional minima in the image. There have been improvements in the original watershed transform in order to reduce its drawbacks. These include using marker-controlled watershed paradigm [23] as well as hierarchical watershed paradigms such as the waterfall algorithm [24]. It must be specify that here the standard framework of watershed transform based on the flooding algorithm is adopted despite to there are other alternative frameworks based on a continuous formulation using topographic distance $[25,26]$; the topological watershed based on discrete geometry tools [27, 28]; graph-based watershed using minimum spanning-tree algorithms $[29,30]$; the power watershed algorithm [31]; the viscous watershed [32]; etc.

With the marker-controlled algorithm, a set of markers imposes the new minima, and the number and position of output regions can be controlled and the over-segmentation problem is reduced. The definition of these markers is not an easy task for the segmentation of the liver, which is a large organ that has an enhanced vessel tree that produces high internal gradients. The manual definition of these markers is inefficient and is not a practical option in clinical environments due to the potential benefits of the algorithm (such as low user interaction) decrease [33]. To deal with problems of this kind, the use of a new variation of the stochastic transform proposed by [34] is carried out in this paper. This variation is necessary because when the original stochastic transform is applied in MR images of the liver, it enhances internal edges with respect to the external edges of the liver, which is not useful for our purpose. The purpose of the new variant of the stochastic watershed proposed in this work is to obtain a more significant probability density function of contours by taking into account the contrast between adjacent regions thanks to a region-based model. Besides presenting this new version of stochastic watershed, another contribution of this work is the combination of pre-processing, marker extraction, and postprocessing filters. This makes possible the liver segmentation of $3 \mathrm{D}$ studies in a fully automated and accurate way and with a low computational cost. These 
features convert the method into a usable tool for clinical purposes.

There have been other liver segmentation algorithms where the watershed transform has been used due to its easy user initialization/interaction, its reasonable computational cost, its intuitiveness, and the accurate results achieved in other casuistic (type of images, organs, etc). In [35], the watershed transform is combined with neural networks to train and tune watershed parameters for liver segmentation purposes. This training algorithm requires manual segmentation, and since the method is only used for 2D image segmentation, the particularities of a 3D volume are not taken into account. In [36], after applying a pre-processing step, the watershed transform is applied but regions with similar intensities may be incorrectly merged due to problems of intensity that are produced by lesions or by illumination that is not uniform.

In our application, the final goal of the segmentation of the liver is to add the $3 \mathrm{D}$ model of the liver into a 3D model with other abdominal organs of a patient previously segmented with own algorithms [37, 38]. This virtual 3D model will be registered and merged thanks to augmented reality algorithms by using an image of the patient that will be taken with an external video camera. The system will be applied in order to place trocars in the patient's body in which the minimum accuracy required is approximately 2 centimetres [39].

The rest of the paper is divided into three sections. The first section describes a technical explanation of the watershed transform, the contribution of this work, and the final method developed for the liver segmentation. The second section explains the datasets used, the training procedure, the optimal parameters, and the results of this new method. The last section describes a discussion of our conclusions and future work.

\section{Image processing methods}

Let $f$ be a grayscale image defined as the mapping

$$
f(\mathbf{x}): \mathrm{E} \rightarrow \mathcal{T},
$$


where $\mathbf{x} \in \mathrm{E}$ is the pixel position in the support space of pixels $\mathrm{E}$, e.g., for $2 \mathrm{D}$ images $\mathrm{E} \subset \mathbb{Z}^{2}$. In the case of valued discrete images, $\mathcal{T}=\left\{t_{\min }, t_{\min }+\right.$ $\left.1, \ldots t_{\max }\right\}$ represents the pixel graylevel. Typically, in 8-bit images, $t_{\min }=0$ and $t_{\max }=255$. Furthermore, let $B(\mathbf{x})$ be a subset of $\mathbb{Z}^{2}$ called flat structuring element (SE) that is centred at point $\mathbf{x}$ with a particular size and shape.

\subsection{Watershed transform using markers}

The watershed transform is a technique based on mathematical morphology for image segmentation [40]. From a morphological point of view, the function $f(\mathbf{x})$ to be segmented, belonging to $\mathbb{Z}^{2} \times \mathbb{Z}$, can be seen as a topographic surface: the lighter the gray value of $f$ at point $\mathbf{x}$, the higher the altitude of the corresponding point $\{\mathbf{x}, f(\mathbf{x})\}$ on the surface. Following this viewpoint, each regional minimum of $f(\mathbf{x})$ represents a region called catchment basin. The purpose of the watershed transform is to flood these basins in order to increase the water level. When two neighbouring basins come into contact with each other, a dam is built at these contact points to prevent the merging between two different basins. At the end, the union of all complete dams constitute the watershed lines, which separates the lakes (catchment basins associated to each

minimum). From a practical viewpoint, watershed transform is computed using fast algorithms based on hierarchical queues [40, 41]. A review of watershed algorithms can be found in [42]. Typically for image segmentation, the gradient of the image is considered as the input image for the watershed transform:

$$
f \mapsto \rho(f) \mapsto W S(\rho(f)),
$$

where the image $W S(\rho(f))$ corresponds to the set of closed contours of the $N$ segmented regions and $\rho(f)$ is the morphological gradient [43]. The number of regions $N$ equals the number of minima of the gradient image.

The result of the watershed can be also considered by a dual representation as an image partition of the support space $\mathrm{E}$ into $N$ connected classes $\mathcal{C}_{n} \subset \mathrm{E}$, 
denoted $\Pi(W S(\rho(f)))$, such that

$$
\begin{aligned}
& \Pi(W S(\rho(f)))=\left\{\mathcal{C}_{n}\right\}_{1 \leq n \leq N} ; \\
& \text { with } \bigcup_{1 \leq n \leq N} \mathcal{C}_{n}=\text { E and } \mathcal{C}_{p} \cap \mathcal{C}_{q}=\emptyset, \forall p \neq q
\end{aligned}
$$

We notice that it is needed that each arc (watershed line) which separates two regions (catchment basin) belongs to one of them. It is well known that without any pre-processing, watershed transform usually leads to an over-segmentation of the image gradient because of the presence of a great number of regional minima due to the noise and the different structures present in images of this type. The classical approach for solving this problem is known as the markercontrolled watershed [40] which involves a reconstruction by erosion $\left(R^{\varepsilon}\right)$ [43] of the gradient image $\rho(f)$ by a marker image $m(\mathbf{x})$ :

$$
W S(\rho(f))_{m(\mathbf{x})}=W S\left(R_{(\rho(f) \wedge m(\mathbf{x}))}^{\varepsilon}(m(\mathbf{x}))\right),
$$

where $m(\mathbf{x})$ is a set of prior markers:

$$
m(\mathbf{x})= \begin{cases}0, & \text { if } \mathbf{x} \text { belongs to a marker } \\ 255, & \text { otherwise }\end{cases}
$$

such that the operation $R_{(\rho \wedge m(\mathbf{x}))}^{\varepsilon}(m(\mathbf{x}))$ imposes the new regional minima of the $f$ gradient, $\rho(f)$, to the pixels set to zero in $m(\mathbf{x})$. Thus, the number of obtained regions equals now the number of connected components of marker image $m(\mathbf{x})$.

\subsection{Stochastic watershed transform}

An appropriate marker definition is essential to obtain a good segmentation result with the marker-controlled watershed. In the stochastic watershed a different strategy is followed due to a stochastic procedure when markers are randomly generated [34]. This arbitrary choice is compensated by the $M$ markedcontrolled watershed realizations of the algorithm in which non-significant fluctuations are filtered out by the stochastic procedure. 
More precisely, stochastic watershed is based on applying the marker-controlled watershed $M$ times to the gradient of the input image, but the markers change randomly in each iteration. Let $\left\{m_{j}(\mathbf{x})\right\}_{j=1}^{M}$ be $M$ sets of $N$ random markers, and let $W S_{j}=W S(\rho(f))_{m_{j}(\mathbf{x})}$ be the j-th output image of the markercontrolled watershed imposed by $m_{j}(\mathbf{x})$. Using this approach, a probability density function of the contours of the image can be obtained with the Parzen method [44] as follows:

$$
\operatorname{pdf}(\mathbf{x})=\frac{1}{M} \sum_{j=0}^{M} W S_{j}(\mathbf{x}) * G(\mathbf{x} ; s),
$$

where $G(\mathbf{x} ; s)$ represents typically a Gaussian kernel of variance $s^{2}$ (in our case, $s=3)$ and mean $\bar{m}(\bar{m}=0)$, that is defined as:

$$
G(\mathbf{x} ; s)=\frac{1}{2 \pi s^{2}} e^{-\left(\frac{\|\mathbf{x}\|^{2}}{2 s^{2}}\right)} .
$$

Besides the pairs of parameters $(\mathrm{M}, \mathrm{N})$, the other variable that can affect the final result of the stochastic watershed algorithm is the procedure for generating the random markers. In the case of random uniform markers, as introduced in [45], the probability density function of contours can be explicitly computed without using $\mathrm{M}$ simulation of markers and corresponding watershed transforms.

\subsubsection{Procedure for generating random markers}

In the basic formulation of stochastic watershed, a uniform distribution in the whole image is used in the marker definition process. Let us consider the problem of liver segmentation tackled in this paper.

Differences in liver regions between adjacent slices are limited and this is the rationale behind our propagative approach of $2 \mathrm{D}$ segmentation. If a good segmentation in one slice is obtained, the resulting mask can be dilated and the perimeter of this dilation can be considered as external marker for the adjacent image (on axial axis) with the certain that all the liver regions will be inside of this external marker. It will help to eliminate adjacent structures close to the liver and with similar graylevel. The random markers (or internal markers) will be generated with a uniform distribution under the constraint that they 
will be inside of the region defined by the previous mask (Fig. 1a). Thus it is guaranteed that the most of the markers will belong to liver regions and the stochastic watershed will not enhance edges of other structures, because no marker will be inside of them.

In the method proposed in this paper, another strategy for generating random markers is also considered in the last marker-controlled watershed applied which will produce the final mask of the liver. The bounding box of the previous mask is divided into a uniform grid, and a random marker is calculated in each region of this grid (Fig. 1b). If the mask is not active (pixels set to zero) in a region of the grid, no marker is generated. This generation procedure will be called as stratified random marker generation. The under-segmentation of neighbourhood regions with similar graylevel is reduced with this variation because markers are better distributed but internal boundaries can be enhanced with this second strategy (because the arbitrary choice is biased). For this reason a uniform-distributed random markers is used first in the generation of the $\operatorname{pdf}(\mathbf{x})$ as it will be explained in section 2.4 and a stratified random markers is used finally in the last iteration of the algorithm when internal gradients are less enhanced.

Both strategies proposed in this paper (uniform and stratified random marker distribution) were applied on the previous mask. The different procedures from the original stochastic watershed [34] were also considered, but the novel procedures proposed in this work are better adapted to the liver segmentation problem.

\subsubsection{Probabilistic partitions from stochastic watershed (PPSW)}

A new paradigm of the stochastic watershed is introduced here to produce a more reliable density of contours. This new stochastic watershed version is needed because using classical stochastic watershed, the liver contour is relatively well defined (Fig. 2c), but the internal boundaries are also enhanced on account of hepatic tree or illumination differences. This is justified since the probability density function (Eq. 5) is extracted directly from the gradi- 


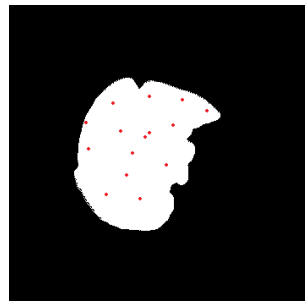

a)



b)

Figure 1: a) Uniform-distributed random markers. b) Stratified random markers (red) and grid zones in which no internal marker is calculated (green).

ent image, which not only contains useful information about the liver edges. This is the reason why the stochastic watershed is not enough selective for the liver segmentation purpose. In order to remove high internal gradients and to keep the external boundaries of the liver enhanced enough, we initially consider the approach suggested in [34] to compute a gradient-like function defined by a trade-off (a convex combination) between the initial gradient and the probability density of contours, i.e.,

$$
\bar{h}_{i}(\mathbf{x})=\alpha \rho(f)_{i}(\mathbf{x})+(1-\alpha) \operatorname{pdf}_{i}(\mathbf{x})
$$

where typically $\alpha=0.5$. However, as can be observed in Fig. 2d, the internal boundaries are still enhanced (with high internal boundaries belonging to adjacent regions with poor contrast). Therefore, a new model based on regional graylevel properties for reducing internal gradients, and keeping the liver edges enhanced, is introduced in the stochastic procedure. The idea is based on the evidence that internal gradients of the original image are not as enhanced as the stochastic watershed produces, so if this regional information of internal gradients can be added to the stochastic watershed procedure, the probability density function will enhance liver edges for segmentation purpose.

According to our approach, $\operatorname{pdf}_{i}(\mathbf{x})$ gives for each pixel $\mathbf{x}$ the probability density estimated from the gradient image which only measures the local energy of the contours. We initially need to calculate from the $\operatorname{pdf}_{i}(\mathbf{x})$ a watershed 


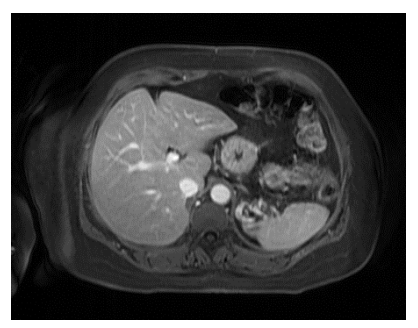

a)

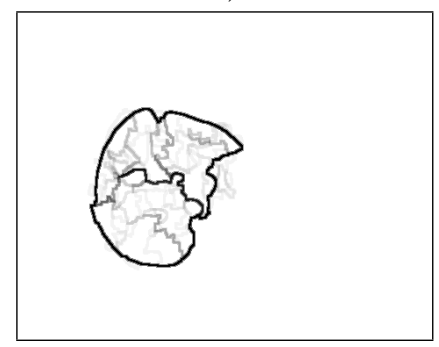

c)

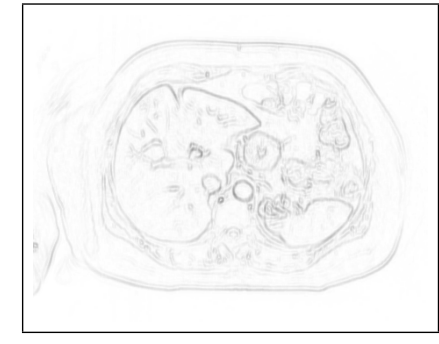

b)



d)

Figure 2: a) Original image. b) Original image gradient. c) Probability density function of image contours $\left(\operatorname{pdf}_{i}(\mathbf{x})\right)$ with $\mathrm{M}=20$ and $\mathrm{N}=15$. d) Probabilistic gradient as proposed in [34], $\bar{h}_{i}(\mathbf{x})$.

transform to obtain an image partition denoted $\Pi_{i}$, i.e.,

$$
\operatorname{pdf}_{i}(\mathbf{x}) \mapsto W S\left(\operatorname{pdf}_{i}\right)(\mathbf{x}) \mapsto \Pi_{i}=\Pi\left(W S\left(\operatorname{pdf}_{i}\right)\right)
$$

Each $n$-connected class $\mathcal{C}_{i, n}$ of the partition $\Pi_{i}$ is then valued with the mean intensity of pixels belonging to this class from the initial image $f_{i}$ in order to construct a "mosaic image", denoted $p_{i}(\mathbf{x})$, and formally defined as

$$
p_{i}(\mathbf{x})=\left\{\mu_{\mathcal{C}_{i, n}}\left(f_{i}\right): \mathbf{x} \in \mathcal{C}_{i, n}\right\}
$$

where $\mu_{\mathcal{C}_{i, n}}\left(f_{i}\right)=1 /\left|\mathcal{C}_{i, n}\right| \sum_{\mathbf{y} \in \mathcal{C}_{i, n}} f_{i}(\mathbf{y})$ is the average of pixel values of slice $f_{i}$ in the connected class $\mathcal{C}_{i, n}$. The morphological gradient of this mean-based simplified model of the image can be interpreted as the regional edgeness energy associated to the probability density function of contours $\operatorname{pdf}_{i}(\mathbf{x})$, i.e.,

$$
\mathcal{E}_{i}^{\text {regional }}(\mathbf{x})=\rho\left(p_{i}(\mathbf{x})\right)
$$


After normalizing this energy to the interval $[0,1]$ both terms can be multiplied, which leads to a regional-adjusted probability density of contours

$$
\widetilde{\operatorname{pdf}}_{i}(\mathbf{x})=\operatorname{pdf}_{i}(\mathbf{x}) \cdot \mathcal{E}_{i}^{\text {regional }}(\mathbf{x})
$$

Nevertheless, we have observed that the results can still be improved by introducing the regional edgeness energy term in each of the $M$ realizations of the stochastic watershed. That is, by integrating the gradient of the meanbased simplified model of each realization instead of directly the watershed lines. Formally, we have

$$
\operatorname{pdf}_{i}^{\text {regional }}(\mathbf{x})=\frac{1}{M} \sum_{j=0}^{M} \rho\left(p_{i, j}(\mathbf{x})\right) * G(\mathbf{x} ; s),
$$

where $p_{i, j}(\mathbf{x})$ is the mosaic image of realization $j$ obtained as:

$$
p_{i, j}(\mathbf{x})=\left\{\mu_{\mathcal{C}_{i, j, n}}\left(f_{i}\right): \mathbf{x} \in \mathcal{C}_{i, j, n}\right\}
$$

from the connected classes $\mathcal{C}_{i, j, n}$ of the partition $\Pi_{i, j}$ obtained from:

$$
\Pi_{i, j}=\Pi_{i}\left(W S_{j}\left(h_{i}\right)\right)
$$

Finally, the probabilistic edgeness function for slice $i$ is defined as the image:

$$
e_{i}(\mathbf{x})=0.5 \rho(f)(\mathbf{x})+0.5 \operatorname{pdf}_{i}^{\text {regional }}(\mathbf{x})
$$

We note that, using the initial probability density function of contours $\operatorname{pdf}_{i}(\mathbf{x})$, each boundary of the output image $W S_{j}$ has the same weight during stochastic integration, Fig. 3a. With the first proposed modification $\widetilde{\operatorname{pdf}}_{i}(\mathbf{x})$, the boundaries associated to a low contrasted regions (internal zones of the liver) are less enhanced since their weights are associated to the contrast. However, the effect is still more notable in the case of the final probabilistic edgeness function $e_{i}(\mathbf{x})$, see Fig. 3c and Fig. 3d.

\subsection{Segmentation algorithm for initial slice}

The first processed slice must undergo a special processing. It is important to carry out a precise segmentation of this first slice because its result will 


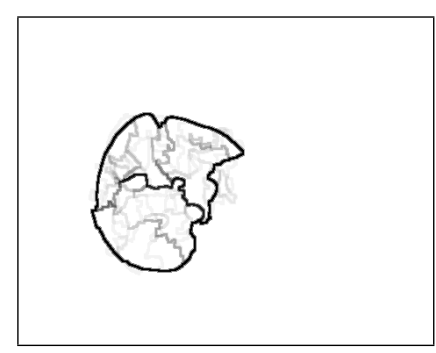

a)

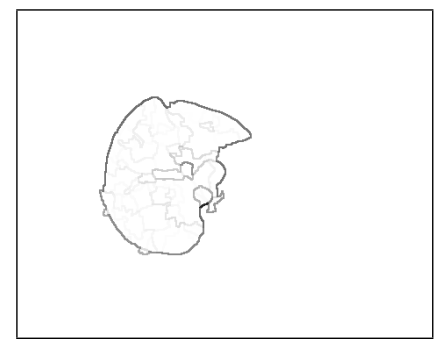

c)

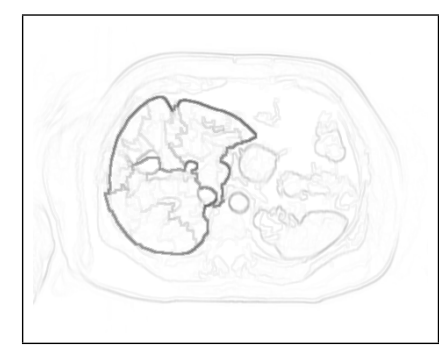

b)

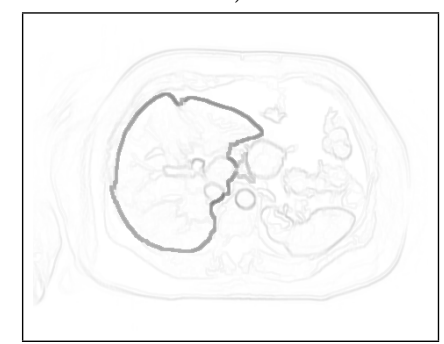

d)

Figure 3: a) Probability density function of image contours $\left(\operatorname{pdf}_{i}(\mathbf{x})\right)$ with $\mathrm{N}=20$. b) Probabilistic gradient as proposed in [34], $\bar{h}_{i}(\mathbf{x})$. c) Regional edgeness energy term, $\operatorname{pdf}_{i}^{\text {regional }}(\mathbf{x})$. d) Final probabilistic edgeness introduced in this paper, $e_{i}(\mathbf{x})$.

be expanded to adjacent slice in order to calculate parameters as explained in section 2.2.1. Manual segmentation is an option for this first slice, but an automatic process based on the marker-controlled watershed has been developed in our case, which is summarized in Fig. 4.

In this initial 2D slice of the 3D image, the liver should appear as large as possible and with a homogeneous graylevel. This slice could be selected manually although in this paper an automatic process to this selection based on the previous assumption on liver features is proposed. First, the graylevel histogram of all the voxels of the whole study is calculated. If the pixels near to zero are excluded, the maximum of the histogram is associated to the liver regions because the liver is the largest organ in the abdominal cavity. Finally, the slice with more pixels whose graylevel is equal to this maximum is selected as the initial slice of the study. With this procedure, in all the processed datasets 
the initial slice selected was appropriate and fulfilled initial restrictions (the liver appears as large as possible and with homogeneous graylevel).

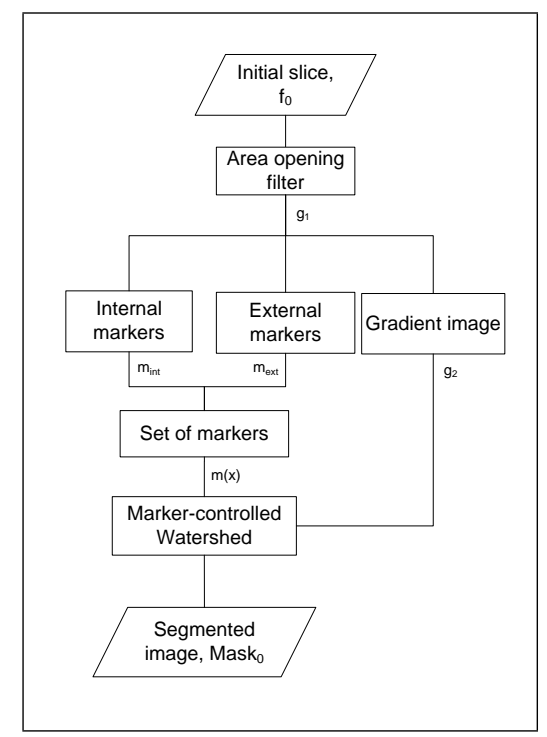

Figure 4: Diagram block of the segmentation procedure of the first slice in the study.

Let $f_{0}$ be the initial $2 \mathrm{D}$ slice to be segment and the goal is obtained a set of markers and a reasonable enhanced gradient for applying the markercontrolled watershed transform. The purpose of the first step is to obtain a well-defined gradient of the liver. If the gradient is calculated on the original slice, i.e., $g_{0}=\rho\left(f_{0}\right)$, our algorithm does not produce the required accuracy in many cases (Fig. 5b). For this reason, an area opening filter is applied to the original slice, resulting in $g_{1}=\gamma_{\lambda}\left(f_{0}\right)$ [43]. This filter reduces the noise and local bright structures (vessels) while the boundaries of the liver are preserved (Fig. $5 c)$.parameter $\lambda$ of this filter corresponds to the area measure. Local bright structures (in our case vessels) with lower area than $\lambda$ will be integrated into local background structures (in our case, liver tissue). The gradient is calculated on the filtered image, $g_{2}=\rho\left(g_{1}\right)$, producing an image where the contour of the liver appears more enhanced and less noisy than in $g_{0}$ (Fig. 5d).

In the next step, before applying the marker-controlled watershed transform, the set of markers is calculated using the output image of the area opening filter 


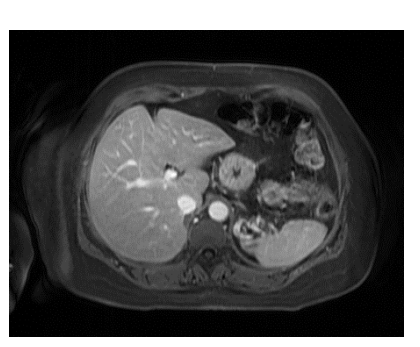

a)



c)

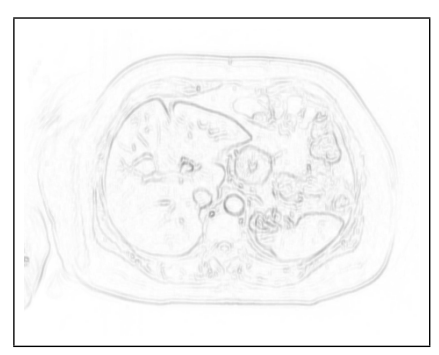

b)

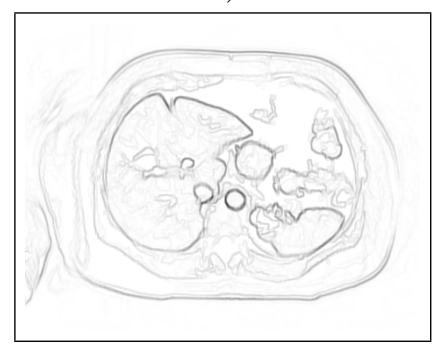

d)

Figure 5: a) Original slice, $f_{0}$. b) Gradient image of original slice, $g_{0}$. c) Area opening filtered image, $g_{1}$. d) Gradient of area opening filter output, $g_{2}$.

as the input of the process. The marker definition not only requires internal markers (that mark the object of interest) but also external markers that are used to constrain the growth of the regions. In this first slice, an external marker image $m_{e x t}$ is obtained as the boundary of the patient's abdomen as previously was proposed in [46].

Internal marker image $\left(m_{\text {int }}\right)$ is obtained from the output image of the area opening filter, $g_{1}$ applying some anatomical restrictions. First, it is sure that the liver will appear on the right part of the image and it will be inside of external marker image, so a sub-image can be calculated (Fig. 6a), $g_{2}$. It is well known that the liver is a structure with higher intensities than other structures in MR images (T1 or T2 weighting) as skin, air or bones. If the accumulated histogram in the complement of this sub-image is calculated, a threshold can be obtained when the $50 \%$ of the pixels are accumulated, to obtain the graylevels belonging to the liver. The pixels with a graylevel smaller than this threshold 
are removed, obtaining image $g_{3}$ (Fig. 6b).

In a second step, in order to remove internal structures (vessels, gallbladder, lesions, etc.) the $10 \%$ of lightest and darkest pixels of $g_{3}$ are deleted and the image is binarized (Fig. 6c). The geodesic distance is calculated and the pixel with the maximum graylevel value is selected as input marker (Fig. 6c, in green).

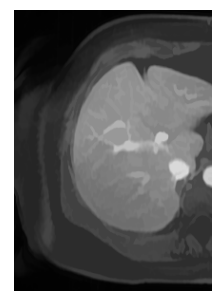

a)

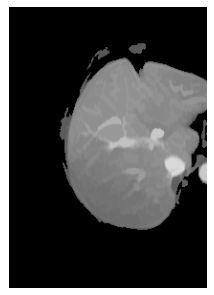

b)

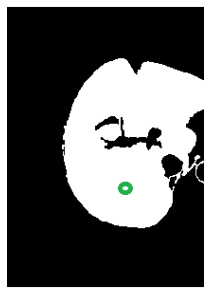

c)

Figure 6: a) sub-image inside the external marker, $m_{\text {ext }}$ (red). b) sub-image after applying the threshold of the accumulated histogram. c) binarized sub-image after removing the $10 \%$ of highest and darkest pixels (the circle in green is the final internal marker selected as the maximun of the geodesic distance).

For watershed segmentation, the final marker image $m(\mathbf{x})$ is given by the union of the internal and the external markers:

$$
m(\mathbf{x})=m_{\text {ext }}(\mathbf{x}) \cup m_{\text {int }}(\mathbf{x}) .
$$

Finally, with this marker image $m(\mathbf{x})$, the marker-controlled watershed transform is applied to image gradient $g_{2}$ (Fig. 7a). Therefore, the output image of the segmentation process of $f_{0}$ is obtained as:

$$
\operatorname{Mask}_{0}(\mathbf{x})=W S\left(g_{2}(\mathbf{x})\right)_{m(\mathbf{x})}
$$

The mask of the liver at this slice is just $\operatorname{Mask}_{0}$ (Fig. $7 \mathrm{~b}$ ).

\subsection{Main segmentation algorithm}

After the segmentation of the first slice, the different steps presented in Fig. 8 are followed to segment the liver into the rest of the slices of the $3 \mathrm{D}$ volume. The current slice and the mask of the previous slice are required as input information. The mask of the initial slice is calculated as was just explained. 




a)

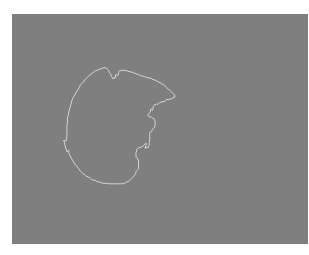

b)

Figure 7: a) Gradient $g_{2}$ and set of markers (red) $m(\mathbf{x})$ for initial slice. b) Result of markercontrolled watershed transform, Mask .

The goal of the different steps of the process is the same as for the first slice: to obtain an appropriate set of markers and a well-defined image to be flooded by the watershed. The algorithm takes advantage of intrinsic parameters of the MR images: the homogeneity in graylevel between neighbouring pixels and the relative co-location of the liver between adjacent slices.

The disadvantages of using directly the marker-controlled watershed transform and the stochastic watershed transform were discussed in Section 1. The algorithm explained for the initial slice not always works correctly in all the images. For that reason, a new paradigm of stochastic watershed introduced in section 2.2 .2 is used.

Let $f_{i}$ be the $\mathrm{i}$-th slice of the whole study, $i \in\left\{-R_{n}, \ldots,-1,1, \ldots R_{k}\right\}$ and let $Q=R_{n}+R_{k}+1$ be the number of the slices of the study. Moreover, let Mask $_{i-1}$ be the liver mask of the previously segmented image on the axial axis. First, an area opening filter is applied, then the gradient of the output image of this slice is calculated, in order to obtain a liver contour image that is more enhanced and less noisy, i.e.,

$$
h_{i}=\rho\left(\gamma_{\lambda}\left(f_{i}\right)\right) .
$$

The new version of the stochastic watershed explained in section 2.2.2 is used to reduced internal gradients. The external marker image $m_{\text {ext }}^{i}$ of each slice corresponds to the external perimeter of a dilation of $M a s k_{i-1}$ as it was explained in the section 2.2.1, i.e.,

$$
m_{e x t}^{i}=\delta_{B_{1} \oplus B_{1}}\left(M_{a s k_{i-1}}\right)-\delta_{B_{1}}\left(M_{a s k_{i-1}}\right) .
$$






Figure 8: Block diagram of main algorithm.

With this external marker image $m_{\text {ext }}^{i}$ and $\mathrm{M}$ uniform-distributed realizations of $\mathrm{N}$ random markers $m_{i n t_{j}}^{i}$, it is obtained $m_{j}^{i}=m_{i n t_{j}}^{i} \cup m_{\text {ext }}^{i}$. These markers are used to calculate the $\operatorname{pdf}_{i}^{\text {regional }}(\mathbf{x})$ of the image contours of slice $i$ and consequently the probabilistic edgeness function, $e_{i}(\mathbf{x})$ defined in Eq. 10.

In the next stage, a final marker-controlled watershed transform is applied to the probabilistic edgeness function. The external marker image is computed as in the previous step: $m_{e x t}^{i}=\delta_{B_{1} \oplus B_{1}}\left(M_{a s k_{i-1}}\right)-\delta_{B_{1}}\left(M_{a s k_{i-1}}\right)$. The internal marker image $m_{i n t}^{i}$ is calculated with a stratified random markers procedure $(7 \times 7$ grid $)$ using the previous result mask, $M a s k_{i-1}$ as it was explained in section 2.2.1. In each region of the grid, an internal random marker is obtained in order to have at least one marker per liver region, which is essential when the liver is broken into several parts (Fig. 1b). A final set of markers is obtained 
by the union of both images: $m_{i}(\mathbf{x})=m_{e x t}^{i}(\mathbf{x}) \cup m_{i n t}^{i}(\mathbf{x})$. Then, a final markercontrolled watershed is applied to obtain a final partition of slice $i$ :

$$
\Pi_{i}^{*}(\mathbf{x})=W S\left(e_{i}(\mathbf{x})\right)_{m_{i}(\mathbf{x})}
$$

At this point, the problem of high internal gradients and the under-segmentation produced if the liver is divided in various regions are solved. However the problem of illumination is only partially solved with the area opening filter (reducing light objects). Hence, a post-processing algorithm is applied to finally correct potential minor segmentation errors.

\subsubsection{Post-processing Classifier}

Let us consider as initial image the mean-valued mosaic image of the partition associated to $\Pi_{i}^{*}(\mathbf{x})$ as it was defined in Eq. 8,

$$
\Pi_{i}^{*}(\mathbf{x}) \mapsto p_{i}^{*}(\mathbf{x}) .
$$

In this mosaic image, the regions belonging to the liver will typically have similar intensity values (under the assumption that the graylevel intensities of the liver have a relatively low variance). However, due to illumination problems, regions belonging to the liver with different graylevel might appear (and the graylevel variance of the liver regions might increase). Therefore, a threshold in the $p_{i}^{*}(\mathbf{x})$ image is not enough to obtain the final mask of the liver. For this reason, a more sophisticated algorithm is needed to minimize the graylevel variance of the regions belonging to the liver while it maximizes the graylevel variance between these regions and external regions that are not liver. This algorithm is detailed in Algorithm 1:

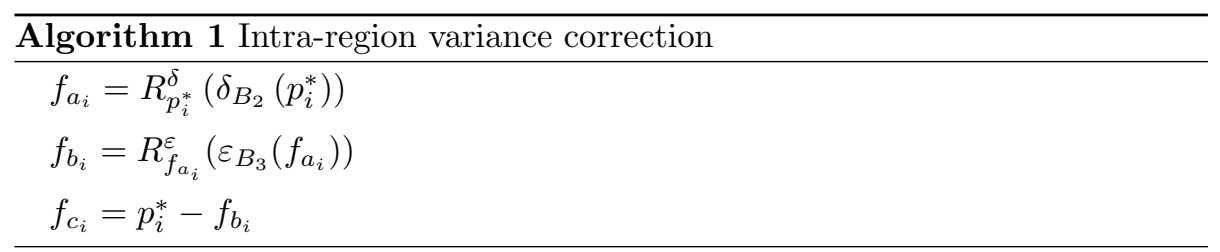

This algorithm has 3 steps: The reconstruction by dilation will reduce light local zones and the reconstruction by erosion will darken local zones with dark 
neighbour zones (typically these zones are in the external boundary of the liver). The result of this algorithm is shown in Fig. 9b. An intensity-based classifier is then applied to the image $f_{c_{i}}(\mathbf{x})$ as follows

$$
f_{d_{i}}(\mathbf{x})=\left\{\begin{aligned}
& 1, \text { if } f_{c_{i}}(\mathbf{x}) \in\left[t_{1}, t_{2}\right], \\
& 1, \quad \text { if } f_{c_{i}}(\mathbf{x}) \in\left[t_{L}, t_{1}\right],\left[t_{2}, t_{U}\right] \\
& \& O V>50 \%, \\
& 0, \quad \text { otherwise. }
\end{aligned}\right.
$$

Thresholds $\left[t_{L}, t_{U}, t_{1}, t_{2}\right]$ are obtained empirically in the training dataset and $O V>50 \%$ means that one of the regions between $\left[t_{L}, t_{1}\right]$ or $\left[t_{2}, t_{U}\right]$ will be added to the mask of the liver if the overlap with the mask of the adjacent slice is more than $50 \%$.
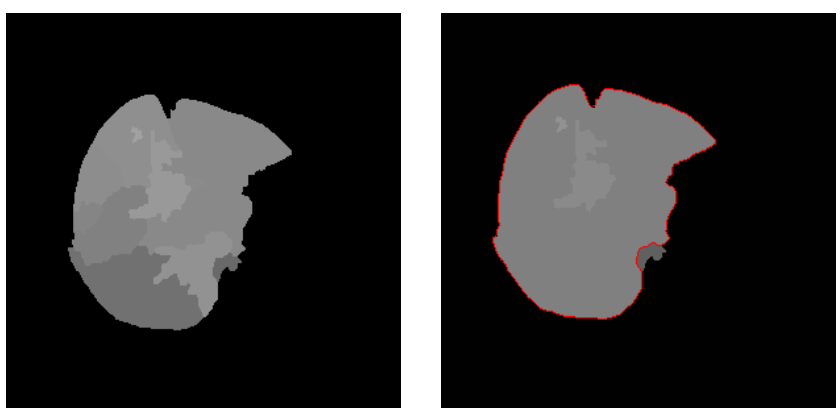

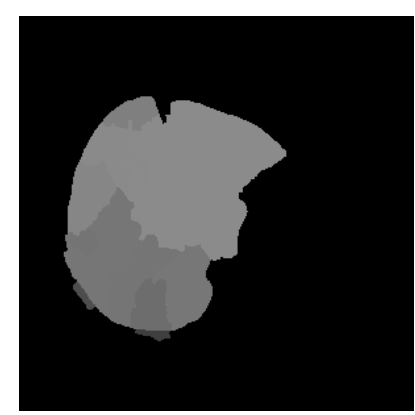

a)

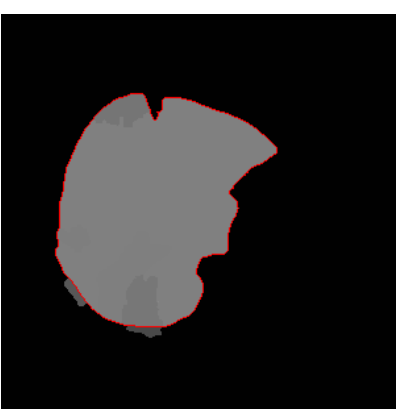

b)

Figure 9: a) $p_{i}^{*}(\mathbf{x})$ and $p_{i+1}^{*}(\mathbf{x})$ images. b) $f_{c_{i}}$ and $f_{c_{i+1}}$ images (in red, $f_{d_{i}}$ and $f_{d_{i+1}}$ contours).

Finally, the mask that is selected as liver is filtered with the Fourier coefficient technique [47] as previously proposed in [9] to smooth the contour, 
obtaining the final mask of the liver for slice $i, \operatorname{Mask}_{i}(\mathbf{x})$.

According to our datasets, a criterion for stopping our algorithm is selected. The algorithm ends when the overlap with the adjacent liver mask is inferior to $50 \%$ or when the mask area is inferior to $3.5 \mathrm{~cm}^{2}$. If the inter-slice distance decreases, the overlap percentage could be more restrictive.

\section{Results}

\subsection{Dataset information and training procedure}

The segmentation method presented in this paper has been evaluated in 17 contrast-enhanced MR studies from Hospital Clínica Benidorm. Table 1 summarizes the technical parameters of all datasets and pathological information. The $\mathrm{x} / \mathrm{y} / \mathrm{z}$ parameter is the voxel spacing; the $\mathrm{w} / \mathrm{h} /$ slices are the number of pixels of the series. The last five series (ID 12-16) were acquired with a Philips Achieva machine (3.0 Tesla), the other datasets with a GE Signa HDX machine (1.5 Tesla). The series with ID: 0-11 were a dynamic sequence acquired when the artery or vein received the contrast agent and the series with ID: 12-16 were a late sequence; that is, they were used to observe the elimination of contrast in lesions.

Dataset 0 was used for tuning the algorithm. This dataset has a combination of the most common problems that are presents in the other studies. Fig. 10 shows some images of this initial dataset where these problems are observable: the vessel enhancement (Fig. 10c), neighbourhood structures with similar graylevel to the liver (Fig. 10a,b), the inter-slice non-uniform illumination (Fig. 10c), and liver visualization into several parts (Fig. 10d).

All the parameters of our method were adjusted with this initial dataset. Empirically, these parameters were changed and different coefficients were calculated. For this purpose, a manual segmentation of this training dataset was carried out by radiologist experts. The final parameters are stored when the coefficients are good enough, and then the rest of datasets are segmented with 
Table 1: Technical and pathological information of the different datasets

\begin{tabular}{|c|c|c|c|}
\hline ID & $\begin{array}{l}\mathrm{x} / \mathrm{y}-\mathrm{z} \\
(\mathrm{mm})\end{array}$ & $\begin{array}{c}(\mathrm{w} / \mathrm{h}) \\
\text { x slices }\end{array}$ & Pathological information \\
\hline 0 (train.) & $0.93-3$ & $512 \times 76$ & Healthy \\
\hline $1-7$ (test) & $\begin{array}{c}{[0.78-1.9} \\
0.88-3]\end{array}$ & $512 \times 59-120$ & Healthy \\
\hline 8 (test) & $0.86-2$ & $512 \times 104$ & Metastasis, seg. I \\
\hline 9 (test) & $0.82-2$ & $512 \times 104$ & Hepatocellular carcinoma, left lobe \\
\hline 10 (test) & $0.85-1.9$ & $512 \times 107$ & multiple metastasis \\
\hline 11 (test) & $0.88-2$ & $512 \times 88$ & Metastasis, right lobe \\
\hline 12 (test) & $0.71-2.5$ & $528 \times 80$ & Hepatocel. carcinoma, 2 lesions seg. VIII \\
\hline 13 (test) & $0.64-2.5$ & $640 \times 80$ & Metastasis, seg. V, IVa, VIII \\
\hline 14 (test) & $0.71-2.5$ & $528 \times 80$ & Focal nodular hyperplasia, seg. VII, VIII \\
\hline 15 (test) & $0.71-2.5$ & $528 \times 80$ & Liver hemangioma \\
\hline 16 (test) & $0.71-2.5$ & $528 \times 80$ & Metastasis, seg. V \\
\hline
\end{tabular}

these parameters. The coefficients and the manual segmentation protocol are the same used in the test dataset.

\subsection{Test Dataset and final parameters}

The performance of our segmentation method was evaluated comparing the automatic liver mask produced by our algorithm with a manual segmentation carried out by four experts (each expert segmented four different series). Moreover, two series of the dataset were selected to be segmented by all them in order to calculate the Inter-expert Cross Correlation coefficient (ICC), obtaining an $I C C=0.622$. This result proves the goodness of this manual segmentation 


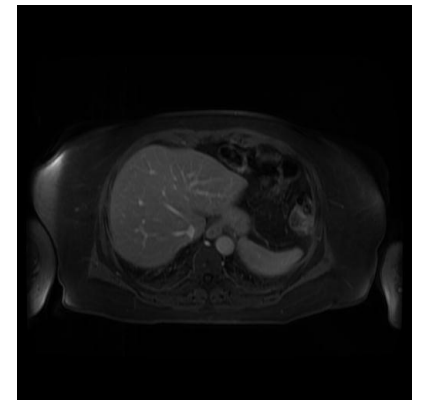

a)

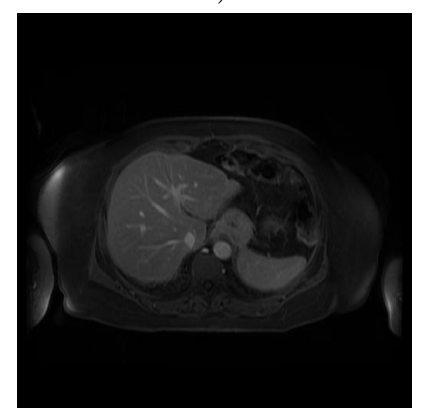

c)

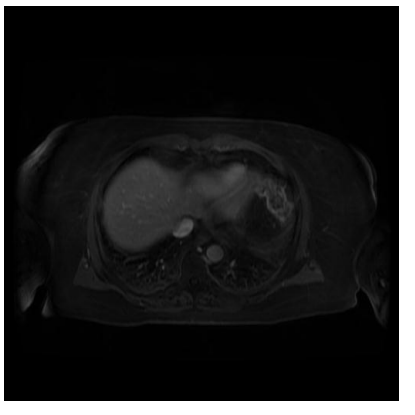

b)

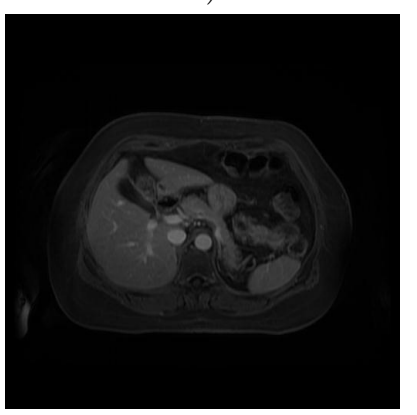

d)

Figure 10: Different problems of the training dataset

because a value greater than 0.61 is considered to be sufficient in order to state that personal differences between the manual segmentation of the experts are not relevant [48].

The coefficients used for the algorithm validation were the Jaccard index and the Hausdorff distance. These coefficients are the most significant for volume comparison and they give complementary information $[49,50]$. Additionally, the Dice Coefficient (DC) is provided for literature comparison (although it provides similar information to Jaccard index). If $X$ is the reference mask and $Y$ is the resulting mask of our segmentation method, these coefficients are defined as:

$$
\begin{gathered}
J C=\frac{|X \cap Y|}{|X \cup Y|} \\
D C=\frac{2 *|X \cap Y|}{|X|+|Y|}
\end{gathered}
$$


The Hausdorff distance is defined as Eq. 16.

$$
d_{H}=\max \left\{\sup _{x \in X} \inf _{y \in Y} d_{\text {Euclid }}(x, y), \sup _{y \in Y} \inf _{x \in X} d_{\text {Euclid }}(x, y)\right\},
$$

where $d_{\text {Euclid }}$ is the Euclidean distance and $(\mathrm{x}, \mathrm{y})$ are two points of both contours $(\mathrm{X}, \mathrm{Y})$ : the manual and the automatic segmentation. A $J C=1$, (or a $D C=1$ ) or a $d_{H}=0$ means that the segmentation is perfect.

The selected parameters (Table 2) were calculated empirically as the optimal parameters for a training dataset and applied to the other studies. The value of $\lambda$ is associated with the minimum area of the liver to be detected and with the internal vessels that the area opening filter integrates in the background. If $\lambda$ increases, vessel contrast is reduced but minimum area to be detected increases. The structuring element used to obtain the external markers is related to the inter-slice distance (a greater distance produces more discontinuities between adjacent slices, so a greater dilation is required to select the liver safely).

The pair of stochastic watershed parameters $(\mathrm{M}, \mathrm{N})$ were chosen based on the experience of [34] and heuristic tests in our training dataset (Fig. 12 and 11). With few realizations $(M=20)$, the $\operatorname{pdf}(\mathbf{x})$ (and the $\left.\operatorname{pdf}^{\text {regional }}(\mathbf{x})\right)$ converges to a stable image (Fig. 11a). The parameter $M$ can be increased $(M=100)$ but the improvement of the results is not significant in this case and computational cost increases considerably (Fig. 11c). The parameter $N$ has a relation to $M$. A high value of $N$ produces many interior regions (Fig. 12c), which is very useful for segmenting a large number of different regions. However, if the goal is the segmentation of the liver tissue (a large organ), the optimal parameter $N$ should be a low value. After different experiments, $N=15$ is applied to all the datasets to ensure the selection of all the liver regions (even if there are not connected (Fig. 12a). If parameters $N$ or $M$ decrease, problems related to gradients definitions could appear as it is appreciated in Fig. 11b and 12b.

The pair of parameters presented in table 2 are very robust against the type of image (only restrictions of size or resolution would be important) for this reason it can be considered as constant values. 
Table 2: Parameters and optimal values

\begin{tabular}{|c|c|}
\hline Filter & Parameters \\
\hline Area opening filter & $\lambda=0.72 \mathrm{~cm}^{2}$ \\
\hline Dilation for external markers & $B_{1}:$ Circular SE, size $=6.8 \mathrm{~cm}^{2}$ \\
\hline $\mathrm{N}$ & 15 \\
\hline $\mathrm{M}$ & 20 \\
\hline
\end{tabular}

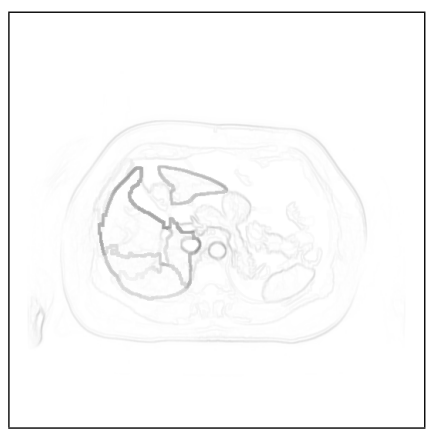

b)

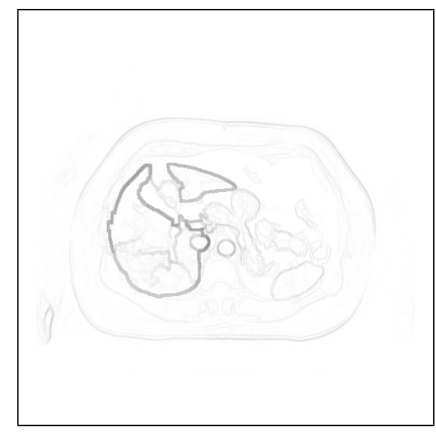

c)

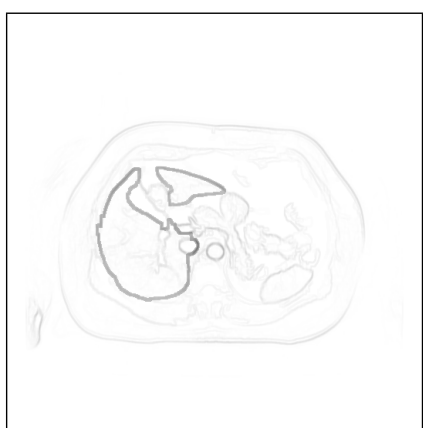

a)

Figure 11: $\operatorname{pdf}(\mathbf{x})$ using different values of parameter $M(N=15)$ : a) $M=5, b) M=20, c)$ $\mathrm{M}=100$.

In the case of the structuring elements $B_{2}$ and $B_{3}$ were selected for reducing bright and dark objects in the final step of our algorithm. Classifier thresholds were selected with the normalized histogram analysis of a set of images, $f_{c_{i}}$, 


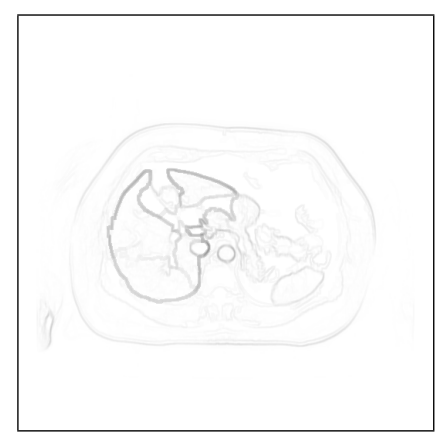

a)

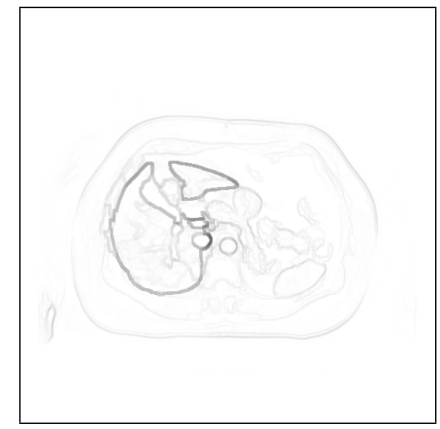

c)

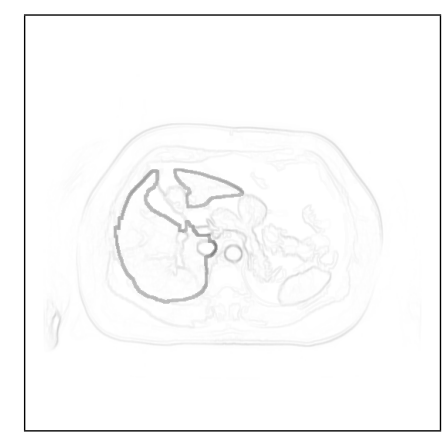

b)

Figure 12: $\operatorname{pdf}(\mathbf{x})$ using different values of parameter $N(M=20)$ : a) $N=5, b) \quad N=15, c)$ $\mathrm{N}=100$.

belonging to different series (some of them are shown in Fig. 15c). This strategy to learn the optimal parameter is justified by the fact that graylevel intensities have a tendency to change between datasets. The global histogram of this set of images is depicted in Fig. 13. Areas between $t_{1}$ and $t_{2}$ fit with liver zones. The areas outside these thresholds cannot always be considered as liver but it is sure that the zones between $\left[t_{1}, t_{L}\right]$ and $\left[t_{2}, t_{U}\right]$ are in the external part of the liver tissue. For this reason, an overlap criterion is used to decide if each of these regions belongs to the liver or not. If it has a $50 \%$ overlap with the previous segmented mask it is considered as liver, otherwise it is labelled as background. Table 3 summarizes the final thresholds of our algorithm applied in all the datasets processed. 
Using the values of tables 2 and 3, the segmentation was carried out in the 17 datasets (training and test datasets) by the method proposed in this paper.

Table 3: Parameters and optimal values of the post-processing step.

\begin{tabular}{|c|c|}
\hline Reconstruction by dilation & $B_{2}$ : Circular SE, size $=10.7 \mathrm{~cm}^{2}$ \\
\hline Reconstruction by erosion & $B_{3}$ : Circular SE, size $=24 \mathrm{~cm}^{2}$ \\
\hline Classifier Thresholds & {$[100-160]$ Liver } \\
$\left(t_{1}-t_{2}\right)$ & {$[70-100,160-190]$ Overlap criteria } \\
$\left(t_{L}-t_{1}, t_{2}-t_{U}\right)$ & {$[0-70,190-255]$ Not Liver } \\
$\left(0-t_{L}, t_{U}-255\right)$ & \\
\hline
\end{tabular}

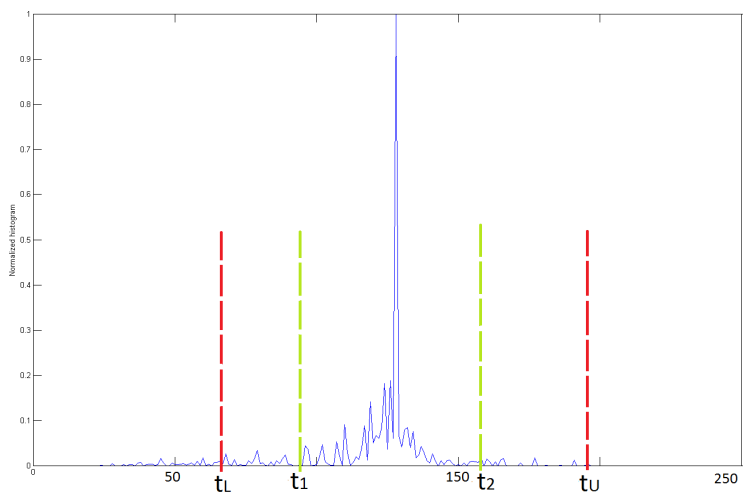

Figure 13: Normalized histogram of the set of images for obtaining optimal thresholds

The values of the validation coefficients are presented in Table 4. All the results were calculated on an Intel core i5 @ $2.80 \mathrm{GHz}$, with a RAM of $2 \mathrm{GHz}$ and Windows 7 (32 bits). The computational cost was about 7 seconds per slice so 7-12 minutes are needed for the segmentation of one dataset.

Fig. 14 shows the segmentation of some initial slices applying the algorithm explained in section 2.3 to different datasets. Several intermediate images of healthy datasets for the process explained in section 2.4 are shown in 
Table 4: Coefficients results

\begin{tabular}{|c||c|c|c||}
\hline Dataset & JC & DC & $d_{H}$ (mm.) \\
\hline \hline Training(ID:0) & 0.9 & 0.95 & 37.19 \\
\hline Healthy(ID:1-8) & $0.9 \pm 0.02$ & $0.95 \pm 0.01$ & $31.61 \pm 4.9$ \\
\hline Unhealthy(ID:9-16) & $0.91 \pm 0.03$ & $0.96 \pm 0.02$ & $35.56 \pm 4.9$ \\
\hline \hline Total(ID:0-16) & $0.91 \pm 0.02$ & $0.95 \pm 0.01$ & $33.58 \pm 6.1$ \\
\hline
\end{tabular}

Fig. 15: the output of the area opening filter $\left(\gamma_{\lambda}\left(f_{i}\right)\right)$, the probabilistic edgeness function $\left(e_{i}(\mathbf{x})\right)$, the illumination-corrected image $\left(f_{c_{i}}\right)$, the initial image $\left(f_{i}\right)$ and the final mask of the liver $\left(M_{a s k_{i}}\right)$ (in red), and the 3D liver model obtained for some studies. Fig. 16 shows the resulting segmentation of some images applying the algorithm explained in section 2.4 to different unhealthy datasets.
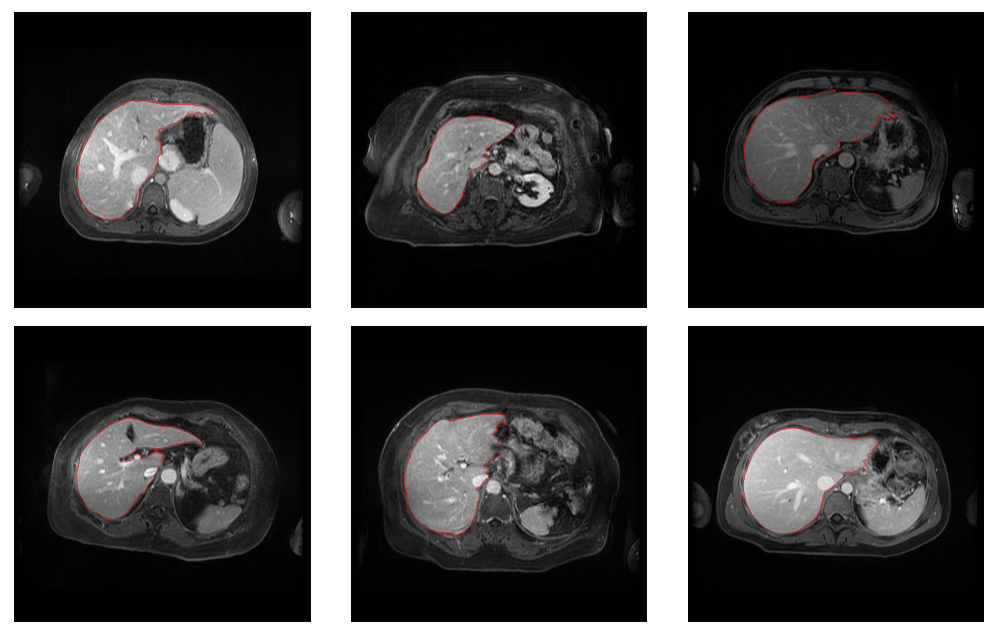

Figure 14: First original slice and segmented mask (in red) for different studies. 

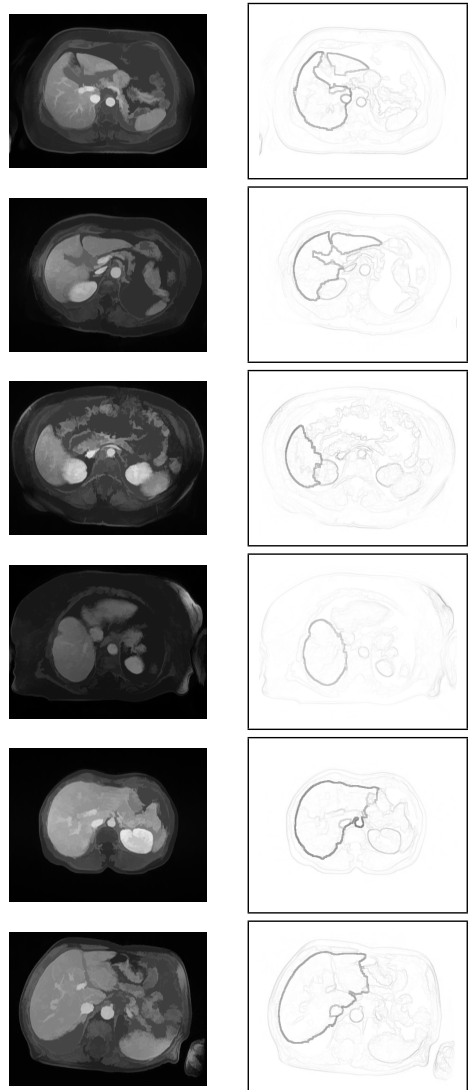

a)
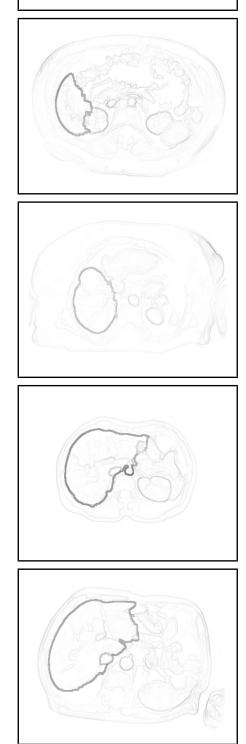

b)
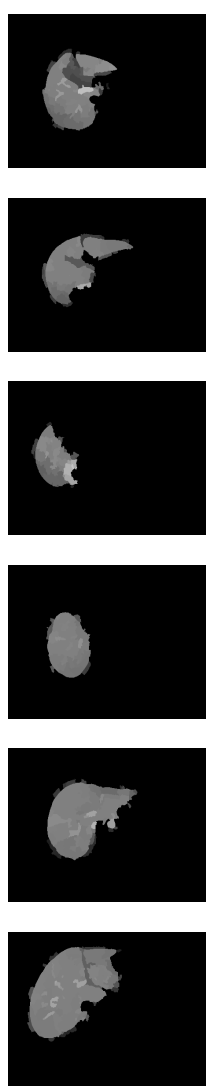

c)
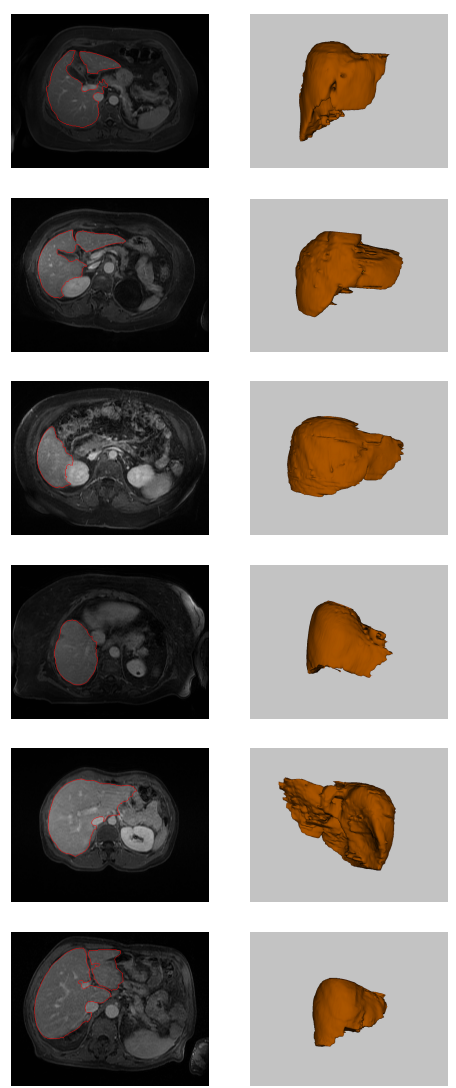

d)
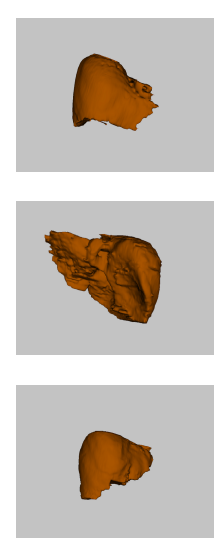

e)

Figure 15: a) Area opening filter output, $\gamma_{\lambda}\left(f_{i}\right)$. b) the probabilistic edgeness function $\left(e_{i}(\mathbf{x})\right)$. c) Corrected image by illumination, $f_{c_{i}}$. d) original image and final mask $\left(f_{i}\right.$, Mask $\left.k_{i}\right)$. e) 3D results of some datasets.

\section{Discussion and Conclusions}

This paper presents a new fully automatic method for liver segmentation in MR images for clinical use. First, a new input image of the watershed transform (instead of the classical gradient) was calculated with a new variant of the original stochastic watershed in order to obtain a probabilistic edgeness function more robust and less noisy than the original gradient. A final marker-controlled watershed was calculated with a set of markers to obtain a final partition (or 

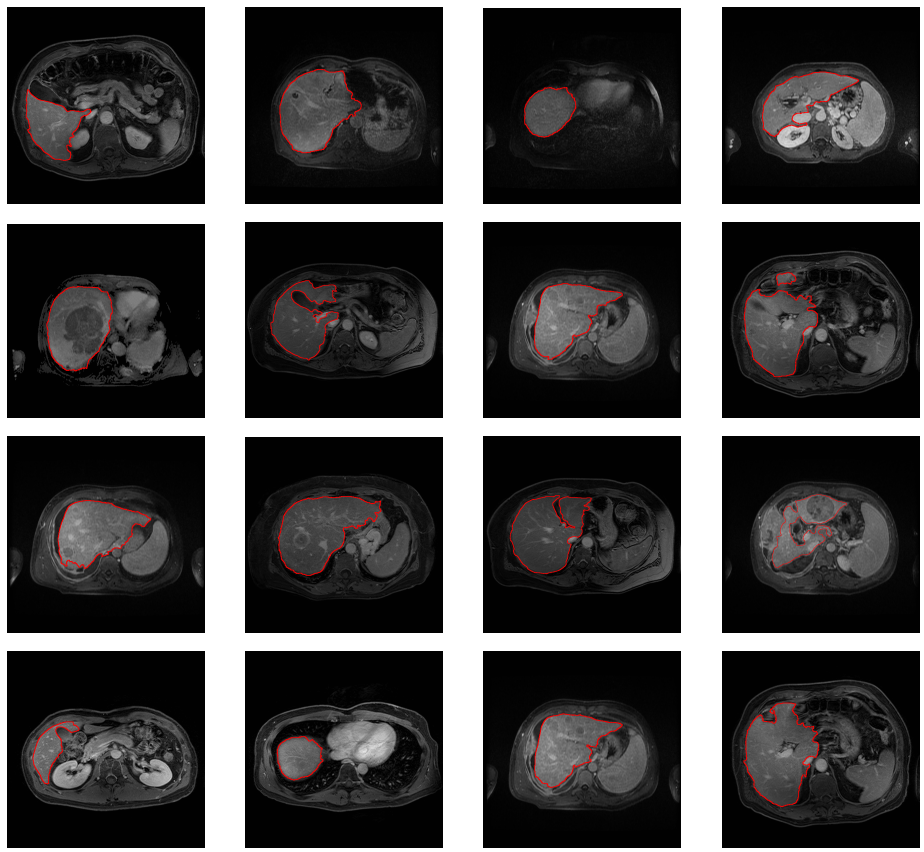

Figure 16: original image and final mask from unhealthy datasets.

"mosaic image") of the liver. Finally, based on the liver graylevel properties and the inter-slice co-location of this organ, a classifier was implemented to obtain the final mask.

The datasets used for the validation were from healthy and unhealthy patients with different properties (spacing, scale, machine, or pathologies). With these considerations, a JC of $0.91 \pm 0.02$ (or a DC of $0.95 \pm 0.01$ ) and the Hausdorff distance of $33.58(6.1) \mathrm{mm}$. demonstrate the accuracy and the robustness (low standard deviation of the coefficient) of the method proposed in this paper.

If a high overlap coefficients and a high Hausdorff distance are obtained, this means that in general a good segmentation is carried out in all the images and some particular problem in some images can produce this high Hausdorff distance. The causes of obtaining a high value of overlap (good segmentation) while a high distance value may be these two: the misclassification is produced in cranial or caudal images and the error propagation is stopped by the algorithm 
when it stops, Fig. 17, or if the misclassification is produced in central images, errors are not spread to adjacent slices. To discover the correct cause of this discrepancy a test was carried out and $10 \%$ of caudal and cranial segmented slices were eliminated and the coefficients were calculated. The results in this new scenario for Hausdorff distance and for Jaccard coefficient improved by $50 \%$ and by $25 \%$ respectively. So the conclusion is the high value of Hausdorff distance had the origin in caudal/cranial images and the worst segmentation is produced in cranial and caudal images. One option to solve these problems is to use additional seed points that can be added manually by the user and that can help to improve the results in these images.

Five of the datasets were acquired in a 3 Teslas MR machine and the others in a 1.5 Teslas MR machine. Table 1 shows that the group of unhealthy patients (where the five 3T studies are included) presents more accurate results despite of being pathological cases and it is probably due to the improvements of MRI hardware. Therefore, it is expected that MRI hardware improvements will improve segmentation results.



(a)



(b)

Figure 17: misclassification: (a) caudal image. (b) cranial image.

Our results were compared with the results of others authors in order to establish our conclusions and future lines of work.

In [1], several methods of liver segmentation are presented and evaluated. These methods have been validated with $10 \mathrm{CT}$ datasets whose spatial resolution is higher than the MRI datasets used in this work. So, the results presented in this paper cannot be compared in a direct manner with the results of these authors but initial conclusions can be extracted from this comparison. In the 
automatic methods, seven authors have a JC in the range (0.91-0.87.5), only two of them have a JC higher than 0.92 , and one author obtains a JC smaller than 0.8. Other coefficients have a similar variability. The main drawbacks of the method that obtained the best results in [1] is that it needed more than 100 liver shapes for the training process and besides that a semi-automatic (and manual) iteration was required in the training step. In general, a high computational cost was required in these methods [15-45 minutes per dataset]. In the iterative methods, JC is better than in the automatic ones but a high computational cost and a hard training or initialization is required.

About MRI methods (table 5), in the fully automatic algorithms only healthy patients were used for the method validation (or fat livers in two cases), i.e, patients with tumors were not used $[20,9,35,22]$. Besides that, two of them used few images for validation $[35,22]$ and other two used only a type of coefficient for the validation procedure $[20,9]$ so a direct comparison with our results is difficult. The runtime of these methods was higher than our method in all the cases. In general, overlap measurements (JC and DC) were better in our method. Regarding to the other three MRI methods presented in table 5 , manual initialization was required $[17,21]$ or few images and qualitative measurements were provided for validating the algorithms [19]. Only one author in table 5 provided overlap (JC or DC) and distance coefficients but few images were used for the validation of this method [22]. Summarizing, to accurately validate a segmentation algorithm both coefficients $\left(d_{H}\right.$ and overlap, i.e, JC or DC) should be calculated [49], so although some authors presented a better Hausdorff distance than our method, we cannot conclude that the accuracy is better. If the high level of automation of our method is taken into account, we can state that our results are promising. A Jaccard coefficient of $0.91 \pm 0.02$ (or $\mathrm{DC}$ of $.95 \pm .01)$ is better than the results obtained by the majority of authors in the studies mentioned above.

The algorithm presented in this paper could be applied directly in the 3D data instead of following a 2D strategy (slice by slice) but some problems have been detected in that case. On the one hand, using the 2D strategy, the external 
Table 5: Results of other authors and main features of their methods. By default runtime is given in minutes per dataset and No. datasets is the number of $3 \mathrm{D}$ datasets used in the validation of each method (2D means that only several $2 \mathrm{D}$ images were used).

\begin{tabular}{|c|c|c|c|c|c|}
\hline Ref. & $\mathrm{JC} / \mathrm{DC}$ & $d H(\mathrm{~mm})$. & Runtime & No. datasets & User-interaction \\
\hline ours & $\begin{aligned} \mathrm{JC} & =0.905(0.027) \\
\mathrm{DC} & =0.95(0.015)\end{aligned}$ & $33.58(6.1)$ & $\begin{array}{c}7-11 \\
7 \mathrm{~s} / \text { image }\end{array}$ & $\begin{array}{l}8 \text { healthy } \\
\text { and } 9 \text { unhealthy }\end{array}$ & fully autom. \\
\hline$[19]$ & - & - & - & 3 images (2D) & $\begin{array}{l}\text { manual segment. } \\
\text { for train. procedure }\end{array}$ \\
\hline$[17]$ & - & - & $8 \mathrm{~s} /$ image & 12 healthy & $\begin{array}{l}\text { initializat. by seeds } \\
\text { low manual work }\end{array}$ \\
\hline$[20]$ & $\begin{array}{l}D C=0.94(0.02) \\
D C=0.89(0.06)\end{array}$ & - & - & $\begin{array}{l}20 \text { fat liver } \\
\text { and } 20 \text { healthy }\end{array}$ & fully autom. \\
\hline [9] & - & $\begin{array}{l}13.51(7.59) \\
20.35(8.66)\end{array}$ & $\begin{array}{l}11.22(2.78) \\
15.37(4.96)\end{array}$ & $\begin{array}{l}10 \text { fat liver } \\
\text { and } 20 \text { healthy }\end{array}$ & $\begin{array}{l}\text { fully autom. } \\
\text { train. requirements }\end{array}$ \\
\hline$[22]$ & $D C=0.92(0.02)$ & $12.74(3.7)$ & $14 \mathrm{~s} /$ image & 6 healthy (2D) & fully autom. \\
\hline [35] & $J C=0.94$ & - & - & 115 healthy (2D) & automatic \\
\hline
\end{tabular}

marker definition is computed as the dilation of the previous segmented mask and the main problem only consists on obtaining an accurate segmentation in the first image. Fig. 14 shows as our algorithm segment the first image with enough accuracy, so the markers chosen with this $2 \mathrm{D}$ strategy contribute to the good performance of our algorithm. On the other hand, if the same algorithm is applied to the 3D data, the 3D marker definition for the background is more complicated. Fig. 18 shows two cases where adjacent structures are misclassified as liver due to the poor gradient between adjacent organs and where the use of patient's skin is used as external marker. However, if the segmentation is carried 
out slice by slice, the external marker is extracted from previous segmented slices where these organs are separated from the liver. When the algorithm segments these problematic slices, the external marker position (a dilation of the previous mask) achieves that the stochastic procedure enhances this poor gradient and improves the performance of the 3D approach.
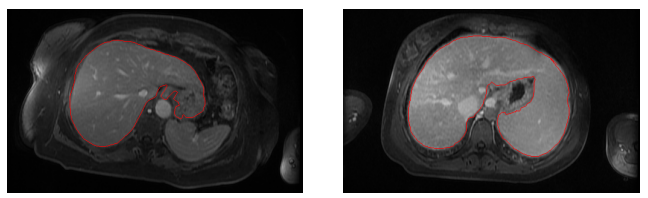

Figure 18: Results in some datasets where a 3D variation of our method is applied

Our segmentation algorithm fulfils the accuracy objective for trocar placement in laparoscopic surgery (the accuracy required for minimally invasive surgery in the insertion of trocars [51]).

\section{Acknowledgments}

This work has been supported by the MITYC under the project NaRALap (ref. TSI-020100-2009-189), partially by the CDTI under the project ONCOTIC (IDI-20101153), by Ministerio de Educación y Ciencia Spain, Project Game Teen (TIN2010-20187) projects Consolider-C (SEJ2006-14301/PSIC), "CIBER of Physiopathology of Obesity and Nutrition, an initiative of ISCIII" and Excellence Research Program PROMETEO (Generalitat Valenciana. Conselleria de Educación, 2008-157). We would like to express our gratitude to the Hospital Clínica Benidorm, for providing the MR datasets and to the radiologist team of Inscanner for the manual segmentation of the MR images.

\section{References}

[1] T. Heimann, B. van Ginneken, M. Styner, Y. Arzhaeva, V. Aurich, C. Bauer, A. Beck, C. Becker, R. Beichel, G. Bekes, F. Bello, G. Binnig, H. Bischof, A. Bornik, P. Cashman, Y. Chi, A. Cordova, B. Dawant, 
M. Fidrich, J. Furst, D. Furukawa, L. Grenacher, J. Hornegger, D. Kainmuller, R. Kitney, H. Kobatake, H. Lamecker, T. Lange, J. Lee, B. Lennon, R. Li, S. Li, H.-P. Meinzer, G. Nemeth, D. Raicu, A.-M. Rau, E. van Rikxoort, M. Rousson, L. Rusko, K. Saddi, G. Schmidt, D. Seghers, A. Shimizu, P. Slagmolen, E. Sorantin, G. Soza, R. Susomboon, J. Waite, A. Wimmer, I. Wolf, Comparison and evaluation of methods for liver segmentation from CT datasets, IEEE Transactions on Medical Imaging 28 (8) (2009) 1251 -1265 .

[2] A. Simpson, P. Dumpuri, W. Jarnagin, M. Miga, Model-assisted imageguided liver surgery using sparse intraoperative data, in: Y. Payan (Ed.), Soft Tissue Biomechanical Modeling for Computer Assisted Surgery, Vol. 11, Springer Berlin Heidelberg, 2012, pp. 7-40.

[3] N. Shevchenko, B. Seidl, J. Schwaiger, M. Markert, T. Lueth, Mimed liver: A planning system for liver surgery, in: Engineering in Medicine and Biology Society, 2010, pp. 1882-1885.

[4] S.-J. Lim, Y.-Y. Jeong, Y.-S. Ho, Automatic liver segmentation for volume measurement in CT images, Journal of Visual Communication and Image Representation 17 (4) (2006) 860 - 875.

[5] L. Ruska, I. Mateka, A. Kriston, Virtual volume resection using multiresolution triangular representation of b-spline surfaces, Computer Methods and Programs in Biomedicine 111 (2) (2013) 315 - 329.

[6] R. Lloréns, V. Naranjo, F. López, M. Alcañiz, Jaw tissues segmentation in dental $3 \mathrm{~d}$ ct images using fuzzy-connectedness and morphological processing, Computer Methods and Programs in Biomedicine 108 (2) (2012) 832 -843 .

[7] G. d'Assignies, C. Kauffmann, Y. Boulanger, M. Bilodeau, V. Vilgrain, G. Soulez, A. Tang, Simultaneous assessment of liver volume and whole liver fat content: a step towards one-stop shop preoperative MRI protocol, European Radiology 21 (2) (2011) 301-309. 
[8] X. Yang, H. C. Yu, Y. Choi, W. Lee, B. Wang, J. Yang, H. Hwang, J. H. Kim, J. Song, B. H. Cho, H. You, A hybrid semi-automatic method for liver segmentation based on level-set methods using multiple seed points, Computer Methods and Programs in Biomedicine 113 (1) (2014) 69 - 79.

[9] O. Gloger, J. Kuhn, A. Stanski, H. Volzke, R. Puls, A fully automatic three-step liver segmentation method on lda-based probability maps for multiple contrast MR images, Magnetic Resonance Imaging 28 (6) (2010) $882-897$.

[10] S. Milko, E. Samset, T. Kadir, Segmentation of the liver in ultrasound: a dynamic texture approach, International Journal of Computer Assisted Radiology and Surgery 3 (1-2) (2008) 143-150.

[11] C. Li, X. Wang, Y. Xia, S. Eberl, Y. Yin, D. D. Feng, Automated pet-guided liver segmentation from low-contrast $\{\mathrm{CT}\}$ volumes using probabilistic atlas, Computer Methods and Programs in Biomedicine 107 (2) (2012) 164 -174 .

[12] H. Dancygier, S. M. Erturk, E. Cay, P. R. Ros, Computed tomography and magnetic resonance imaging, in: Clinical Hepatology, Springer Berlin Heidelberg, 2010, pp. 405-423.

[13] S. M. Hussain, R. C. Semelka, Hepatic imaging: Comparison of modalities, Radiologic Clinics of North America 43 (5) (2005) 929-947.

[14] N. C. Balci, A. S. Befeler, P. Leiva, T. K. Pilgram, N. Havlioglu, Imaging of liver disease: Comparison between quadruple-phase multidetector computed tomography and magnetic resonance imaging, Journal of Gastroenterology and Hepatology 23 (10) (2008) 1520-1527.

[15] A. Hassan, R. Al-Ajami, K. Dashti, M. Abdoelmoneum, Sixty-four multislice computed tomography and magnetic resonance imaging in evaluation of hepatic focal lesions, The Egyptian Journal of Radiology and Nuclear Medicine 42 (2) (2011) $101-110$. 
[16] S. Phongkitkarun, T. Srianujata, J. Jatchavala, Supplement value of magnetic resonance imaging in small hepatic lesion detected on routine computed tomography, Journal of the Medical Association of Thailand 92 (5) (2009) 677-686.

[17] G. Chen, L. Gu, L. Qian, J. Xu, An improved level set for liver segmentation and perfusion analysis in MRIs, IEEE Transactions on Information Technology in Biomedicine 13 (1) (2009) $94-103$.

[18] Z. Yuan, Y. Wang, J. Yang, Y. Liu, A novel automatic liver segmentation technique for MR images, in: International Congress on Image and Signal Processing, Vol. 3, 2010, pp. $1282-1286$.

[19] K. Cheng, L. Gu, J. Xu, A novel shape prior based level set method for liver segmentation from MR images, in: International Conference on Information Technology and Applications in Biomedicine, 2008, pp. $144-147$.

[20] O. Gloger, K. Toennies, J.-P. Kuehn, Fully automatic liver volumetry using 3D level set segmentation for differentiated liver tissue types in multiple contrast MR datasets, in: Image Analysis, Vol. 6688, Springer Berlin / Heidelberg, 2011, pp. 512-523.

[21] C. P. Dueñas, P. M. Gonzalez, M. del Carmen Tobar, J. M. Poncela, F. J. Sanguino, G. Asensio, E. Santos, Automatic method to segment the liver on multi-phase MRI, in: Proceedings of the Computer Assisted Radiology and Surgery 22nd International Congress and Exhibition, CARS 2008, Springer Verlag, New York, EEUU, 2008, pp. 404- 406.

[22] E. Goceri, M. Unlu, C. Guzelis, O. Dicle, An automatic level set based liver segmentation from MRI data sets, in: 3rd International Conference on Image Processing Theory, Tools and Applications (IPTA), 2012, pp. 192-197.

[23] F. Meyer, S. Beucher, Morphological segmentation, Journal of Visual Communication and Image Representation 1 (1) (1990) 21 - 46. 
[24] S. Beucher, Watershed, hierarchical segmentation and waterfall algorithm, in: PROC. of Mathematical Morphology and its Applications to Image Processing, Kluwer, 1994, pp. 69-76.

[25] F. Meyer, Topographic distance and watershed lines, Signal Processing 38 (1994) 113-125.

[26] L. Najman, M. Schmitt, Watershed of a continuous function, Signal Processing 38 (1994) 99-112.

[27] M. Couprie, G. Bertrand, Topological gray-scale watershed transform, in: PROC. of SPIE Vision Geometry V, volume 3168,, 1997, p. 136146.

[28] G. Bertrand, On topological watersheds, Journal of Mathematical Imaging and Vision 22 (2005) 217-230.

[29] F. Meyer, Minimum spanning forests for morphological segmentation, in: PROC. of Mathematical Morphology and its Applications to Image Processing, Kluwer, 1994, pp. 77-84.

[30] J. Cousty, G. Bertrand, L. Najman, M. Couprie, Minimum spanning forests and the drop of water principle, IEEE Transactions on Pattern Analysis and Machine Intelligence 31 (2009) 1362-1374.

[31] C. Couprie, L. Grady, L. Najman, H. Talbot, Power watershed: A unifying graph-based optimization framework, IEEE Transactions on Pattern Analysis and Machine Intelligence 33 (2011) 1384 - 1399.

[32] C. Vachier, F. Meyer, The viscous watershed transform, J. Math. Imaging Vis. 22 (2005) 251-267.

[33] R. J. Lapeer, A. C. Tan, R. Aldridge, A combined approach to 3D medical image segmentation using marker-based watersheds and active contours: the active watershed method, in: PROC. Medical Image Understanding and Analysis, 2002, pp. 165-168. 
[34] J. Angulo, D. Jeulin, Stochastic watershed segmentation, in: PROC. of the 8th International Symposium on Mathematical Morphology, 2007, pp. 265-276.

[35] H. Masoumi, A. Behrad, M. A. Pourmina, A. Roosta, Automatic liver segmentation in MRI images using an iterative watershed algorithm and artificial neural network, Biomedical Signal Processing and Control 7 (5) (2012) $429-437$.

[36] P. Kim, Y. Lee, Y. Jung, J. Cho, M. Kim, Liver extraction in the abdominal CT image by watershed segmentation algorithm, in: PROC. of World Congress on Medical Physics and Biomedical Engineering, Vol. 14, Springer Berlin Heidelberg, 2007, pp. 2563-2566.

[37] F. López-Mir, V. Naranjo, J. Angulo, E. Villanueva, M. Alcañiz, S. LópezCelada, Aorta segmentation using the watershed algorithm for an augmented reality system in laparoscopic surgery, in: 18th IEEE International Conference on Image Processing, 2011, pp. 2649 -2652.

[38] F. López-Mir, V. Naranjo, J. J. Fuertes, M. Alcañiz, J. Bueno, E. Pareja, Design and validation of an augmented reality system for laparoscopic surgery in a real environment, BioMed Research International 2013 (2013) $1-12$.

[39] J. J. Fuertes, F. López-Mir, V. Naranjo, E. Villanueva, , M. Ortega, M. Alcañiz, Augmented reality system for keyhole surgery performance and accuracy validation, in: PROC. of International Conference on Computer Graphics Theory and Applications, SciTePress - Science and Technology, 2011, pp. 273-279.

[40] S. Beucher, F. Meyer, The morphological approach to segmentation: the watershed transformation. Mathematical morphology in image processing., Optical Engineering 34 (1993) 433-481. 
[41] P. Vincent, L. Soille, Watersheds in digital spaces: An efficient algorithm based on immersion simulations, IEEE PAMI, 199113 (6) (1991) 583-598.

[42] J. Roerdink, A. Meijster, The watershed transform: Definitions, algorithms and parallelization strategies, FUNDINF: Fundamenta Informatica 41.

[43] P. Soille, Morphological Image Analysis, Vol. Second edition, Springer, 2002.

[44] R. O. Duda, P. E. Hart, Pattern Classification and Scene Analysis, John Willey \& Sons, New Yotk, 1973.

[45] F. Meyer, J. Stawiaski, A stochastic evaluation of the contour strength, in: Pattern Recognition, Vol. 6376 of Lecture Notes in Computer Science, Springer Berlin Heidelberg, 2010, pp. 513-522.

[46] P. Campadelli, E. Casiraghi, A. Esposito, Liver segmentation from computed tomography scans: A survey and a new algorithm, Artificial Intelligence in Medicine 45 (2009) 185-196.

[47] C. T. Zahn, R. Z. Roskies, Fourier descriptors for plane closed curves, IEEE transactions on computers c-21 (3) (1972) 269-281.

[48] M. Kramer, A. Feinstien, The biostatistics of concordance, Clinical biostatistics January (1981) 111-123.

[49] M. Lago, F. Martínez-Martínez, R. M.J., C. Monserrat, M. Alcañiz, A study about coefficients to estimate the error in biomechanical models used to virtually simulate the organ behaviors, in: Studies in Health Technology and Informatics, Vol. 173, 2012, pp. 250-256.

[50] F. Martínez-Martínez, M. Rupérez, J. Martín-Guerrero, C. Monserrat, M. Lago, E. Pareja, S. Brugger, R. López-Andújar, Estimation of the elastic parameters of human liver biomechanical models by means of medical images and evolutionary computation, Computer Methods and Programs in Biomedicine 111 (3) (2013) 537 - 549. 
[51] M. Feuerstein, Augmented reality in laparoscopic surgery. new concepts for intraoperative multimodal imaging, Ph.D. thesis, Fakultät für Informatik. Technische Universität München. (2007). 